\title{
Transient duodenal erosions in association with superior mesenteric ischemia
}

\author{
D.G. MACINTOSH, MD, D.J. LedDIN, MB, MRCPI, FRCPC
}

ABSTRACT: A 41-year-old male was seen with complaints of periumbilical pain and weight loss. Physical examination, radiological and colonoscopic examinations were unremarkable. Upper endoscopy revealed aphthous ulcers of the antrum and duodenum. At repeat endoscopy two weeks later the erosions had spontaneously healed. Mesenteric angiography revealed occlusion of the superior mesenteric artery and an aberrant right hepatic artery arising distal to the superior mesenteric stenosis. A surgical revascularization procedure was performed at which time occlusion of the inferior mesenteric artery was documented. The patient is now 18 months post surgery and entirely asymptomatic. Can J Gastroenterol 1989;3(1):29.33

Key Words: Aberrant hepatic artery, Aphthous ulceration, Gastroduodenitis, Mesenteric ischemia

\section{Erosions duodénales passagères associées à l'ischémie mésentérique supérieure}

RESUME: Le patient de 41 ans se plaint de douleurs péri-ombilicales et d'une perte de poids. Les examens physique, radiologique et colonoscopique n'indiquent rien de particulier. Une endoscopie supérieure révèle des ulcères aphteux de l'antre et du duodénum. D'après une endoscopie effectuée deux semaines plus tard, les érosions se sont guéries spontanément. Une angiographie mésentérique révèle une occlusion de l'ar tère mésentérique supérieure et une ar tère hépatique droite aberrante en aval de la sténose mésentérique supérieure. Une revascularisation chirurgicale confirme quil y avait bien occlusion de l'artère mésentérique inférieure. Dix-huit mois après l'intervention, le patient est totalement asymptomatique.

Division of Gastroenterology, Health Sciences Centre, Memorial University, St John's, Newfoundland Correspondence and reprints: Dr D J. Leddin, Faculty of Medicine, Memorial University of

Newfoundland, St John's. Newfoundland A IB $3 V_{6}$

Received for publication October 1, 1988. Accepted November 1, 1988
A 41-YEAR-OLD MALE WASREFERRED for investigation of abdominal pain of four months duration. The pain was described as severe and cramping, and was located in the periumbilical region. Partial relief was obtained by bending forward at the waist. There was no clear relationship between development of the pain and eating. On occasion the pain would wake him at night. Typically each episode would last 2 or $3 \mathrm{~h}$. There was associated intermittent nausea and weight loss of $4 \mathrm{~kg}$ in the preceding four months. The patient denied symptoms of mouth ulceration, arthritis or skin rash.

Past medical history was unremarkable except for an appendectomy 20 years previously. The patient's father had died at age 48 of a myocardial infarction. The patient worked as a data consultant and had recently separated from his wife. He smoked one package of cigarettes per day but did not drink alcohol. He was not taking any medications. The systemic enquiry was negative.

On examination the patient was in no distress. Blood pressure was 135/75 $\mathrm{mmHg}$, pulse 84 beats $/ \mathrm{min}$ and regular, and respiratory rate was $15 / \mathrm{min}$. Exam- 
ination of the head and neck was normal, as was that of the cardiovascular and respiratory systems. There were no xanthalasmas. No abnormalities were detected on abdominal or rectal examination.

The initial clinical impression was that of Crohn's disease of the small intestine with intermittent obstructive symptoms.

\section{INVESTIGATIONS}

Hemoglobin was $140 \mathrm{~g} / \mathrm{L}$ (normal 140 to 180 ), white cell count $8.7 \times 10^{9} / \mathrm{L}$ (normal 4.8 to 10.8 ) with a normal differential. Erythrocyte sedimentation rate was $5 \mathrm{~mm} / \mathrm{h}$ (normal 0 to 22 ). Serum electrolytes, urea and creatinine were normal. Serum cholesterol was $5.63 \mathrm{mmol} / \mathrm{L}$ (normal 4.16 to 6.76 ) and serum triglyceride $1.73 \mathrm{mmol} / \mathrm{L}$ ( normal 0.45 to 2.20 ).

A barium enema which had been done prior to consultation was reviewed and found to be normal. An upper GI series and small bowel follow-through were performed. The radiologist felt that this was suspicious for Crohn's disease of the proximal ileum, however, a subsequent small bowel enema showed no abnormalities. A colonoscopy was performed and was unremarkable, the ileum was not visualized.

The patient continued to complain of pain, now occurring after each meal, and fur ther weight loss. Although the symp- toms did not sound gastric or duodenal in origin, an upper endoscopy was performed. Multiple antral erosions and patchy aphthous-like duodenal erosions were seen. These were thought to be compatible with Crohn's disease. Biopsies and brushings were taken.

The biopsies revealed nonspecific inflammation and a repeat endoscopy was performed two weeks later in order to obtain further tissue for histological examination. At this time, unexpectedly, the stomach and duodenum were entirely normal. The patient had not been treated.

A computerized tomography scan was performed to rule out pancreatic and retroperitoneal disease and this revealed calcification of the abdominal aor ta but no other abnormality. The diagnosis became clear. The patient was promptly re-examined and epigastric and right femoral bruits were detected.

A flush aor togram and selective mesenteric arteriograms with subtraction films were carried out. The flush aor togram revealed normal filling of the celiac artery but a significant stenotic lesion was present at the origin of the superior mesenteric artery (SMA). The inferior mesenteric artery (IMA) was not visualized.

A selective celiac axis arteriogram revealed normal filling of the common hepatic, left gastric and splenic arteries (Figures 1 and 2). The common hepatic artery gave rise only to a left hepatic artery. In the delayed films the SMA was seen to fill via the pancreaticoduodenal arcade. The right hepatic artery originated from the SMA distal to the stenotic lesion (Figures 1 and 2). The SMA itself could not be selectively catheterized. Severe stenosis of the left iliac artery was also seen. The finding of retrograde filling of the SMA circulation via the celiac axis was strongly supportive of SMA occlusion since bloodflow through a patent SMA would normally dilute any dye entering the SMA by collaterals.

An SMA bypass was performed. At operation the angiographic findings were confirmed and in addition the IMA was found to be occluded. Following surgery the patient was able to eat without any pain whatsoever. He quickly regained the lost weight. Eighteen months after surgery the patient was well from the gastrointestinal point of view; however he recently suffered a myocardial infarction and is presently recovering.

\section{DISCUSSION}

This 41-year-old man presented with nonspecific abdominal pain and bloating. At presentation there was no clear relationship between eating and devel-

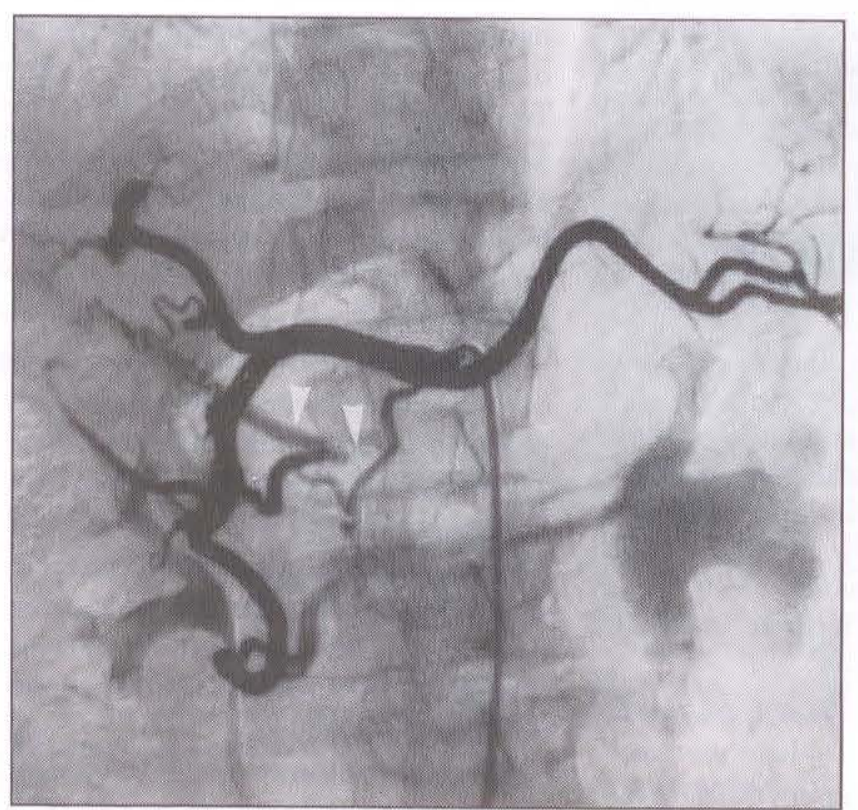

Figure 1) Selective celiac artery catheterization showing retrograde filling of the superior mesenteric artery which was occluded at its origin (arrow) and which gave rise to an aberrant right hepatic artery (arrow)

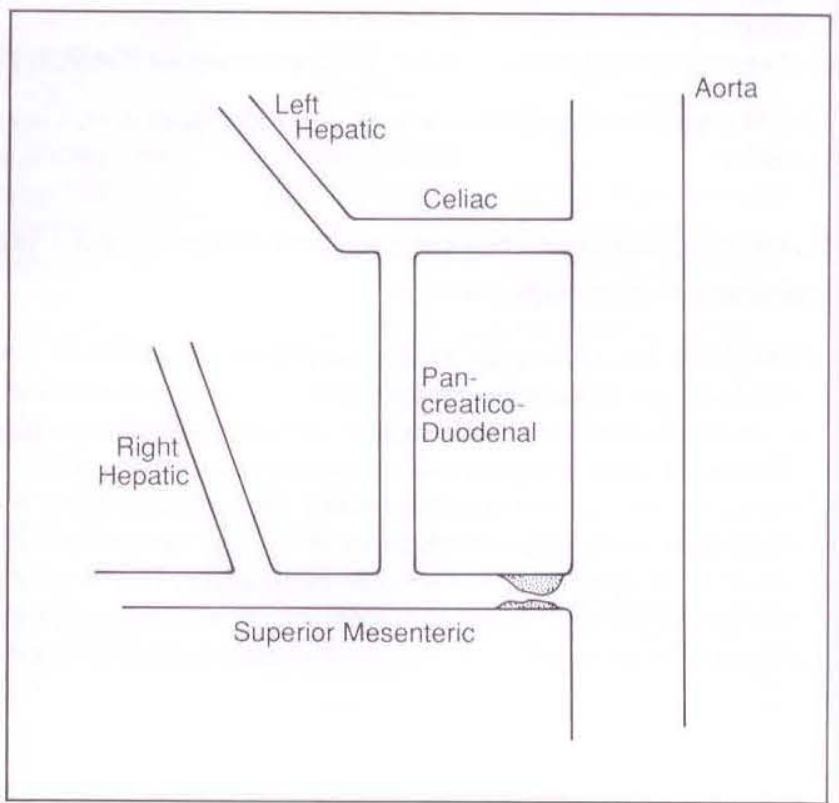

Figure 2) Schematic diagram of angiographic findings showing the ortgin of the aberrant right hepatic artery distal to the superior mesenteric artery occlusion 
opment of the pain. In fact, he was occasionally awoken from sleep by abdominal cramping. Physical examination did not reveal any abnormalities. The clinical impression was of Crohn's disease. Initial radiologic investigations were thought to be compatible with Crohn's disease of the small intestine but this was not supported by subsequent radiological and colonoscopic examinations.

As time progressed the relationship between the pain and eating became more obvious and significant weight loss clearly indicated an organic cause for the patient's symptoms. Endoscopy revealed antral erosions and aphthous ulceration of the duodenum. This again suggested that the diagnosis was inflammatory bowel disease. However, a repeat endoscopy was entirely unremarkable.

At this point the diagnosis could have been made but it was not until a computerized tomography scan of the abdomen revealed aortic calcification that the reason for the patient's symptoms became obvious. Abdominal bruits were noted on re-examination and angiography documented severe stenosis of SMA with an anomalous origin of the right hepatic artery. The IMA appeared to be occluded and this was confirmed at surgery. SMA bypass was performed with prompt resolution of symptoms and a return of normal weight. The patient is currently asymptomatic.

In this patient all disease processes other than mesenteric ischemia were excluded by the intensive investigation to which he was subjected. Although the patient continued to smoke he did not ingest any alcohol or nonsteroidal antiinflammatory drugs. The authors are confident, therefore, that the diagnosis of antral and duodenal erosions secondary to superior mesenteric ischemia was correct.

Under normal conditions, the splanchnic circulation receives approximately $25 \%$ of cardiac output. Of this blood flow $75 \%$ is directed to the mucosa $(1,2)$. The anatomical arrangement of the villus microcirculation allows a countercurrent exchange of oxygen between the afferent arteriole and the efferent venule (3). This may short circuit oxygen delivery to the villus tip and render it susceptible to ischemia. Countercurrent ex- change is especially important during low flow states as may be seen in intestinal ischemia (3).

The metabolic work associated with digestion and absorption considerably increases mucosal oxygen requirements and under normal circumstances mucosal blood flow increases by over $50 \%$ post prandially (4). If mucosal oxygen delivery is inadequate for oxygen requirements ischemia will result. The transient erosions in the present patient probably occurred as a result of the inability of the atheromatous splanchnic circulation to meet mucosal post prandial metabolic requirements.

Atherosclerosis of mesenteric vessels accounts for $90 \%$ of cases of chronic mesenteric ischemia. Predisposing factors include old age, hypertension, diabetes and cigarette smoking. Digitalis has been shown to alter the reactivity of the mesenteric vessels and may aggravate the situation (5).

Chronic mesenteric ischemic syndromes occur less frequently than acute syndromes because of the potential for development of extensive collateral flow in the mesenteric circulation. It is likely that the congenitally aberrant right hepatic artery contributed to the problem in the present patient. It can reasonably be assumed that blood supply to the small intestine and colon in this patient was supplied almost entirely through collateral circulation via the pancreaticoduodenal and iliac arcades. Under normal circumstances blood flow to the SMA and IMA is approximately $900 \mathrm{~mL} / \mathrm{min}$ (2). Blood flow to the right lobe of the liver is approximately $200 \mathrm{~mL} / \mathrm{min}$ (4). The aberrant right hepatic artery may, therefore, have been stealing as much as $20 \%$ of the collateral blood flow.

The history of the present patient is highly suggestive of chronic mesenteric ischemia. Patients with mesenteric angina typically complain of abdominal pain which occurs 15 mins to $1 \mathrm{~h}$ after meals and lasts 2 to $3 \mathrm{~h}$. However, as in this case, the relationship between increased metabolic activity of the intestine post prandially and the development of pain may not be clear. This patient had occasional nocturnal pain and it is difficult to ascribe this to increased metabolic activity of the intestine. The pain is usually located in the upper abdomen or periumbilical area and is described as a dull ache. Bending forward may relieve the pain as it did in the present case (6). The intensity of pain may increase with progression of disease and the severity may correlate with the amount of food ingested and this often leads to avoidance of food and subsequent weight loss. Diarrhea and steatorrhea have been reported (7).

Physical examination may be unremarkable. Signs of weight loss and peripheral vascular disease may be found. The presence or absence of an abdominal bruit is not pathognomonic (8). Laboratory findings are nonspecific.

Making the diagnosis of intestinal angina can be difficult as evidenced by the fact that many patients with acute intestinal infarction have had preceding symptoms of abdominal angina (8). The importance of a good history and physical examination is paramount. If the epigastric bruit and the right femoral bruit, finally detected in this patient, had been detected at presentation the diagnosis may have been made sooner.

Since there is no specific diagnostic test for mesenteric ischemia, conditions which may simulate mesenteric ischemia such as peptic ulcer disease, cholelithiasis, pancreatitis and malignancy must be ruled out. When appropriate studies are normal in a patient with weight loss and abdominal pain, angiography should be considered. Unfortunately, angiography alone cannot confirm the diagnosis. Many asymptomatic elderly patients have significant splanchnic vessel lesions (9). It is necessary, therefore, to apply careful clinical judgement as to whether angiography should be performed.

Erosive duodenitis may occur in association with a wide variety of pathologic processes such as ingestion or irritants, lymphoma, amyloidosis and granulomatous disease. None of these entities were found in the present patient. In particular, there was no ingestion of nonsteroidal anti-inflammatory agents or alcohol. Erosive gastroduodenitis has also been reported in association with vasculitis $(10,11)$, mesenteric atheromatous embolization and occlusive mesenteric ischemia.

Atheromatous embolization with oc- 
clusion of arterial vessels by cholesterol emboli is a well recognized cause of renal failure and cerebrovascular infarction (12). Gastrointestinal involvement also occurs. Atheromatous embolization has been implicated in the development of gastric infarction, hemorrhagic gastritis, gastric and duodenal erosions, small intestinal necrosis, pancreatitis and colonic hemorrhage (13-18). The embolic process may involve single or multiple organs and the course may fluctuate with time.

Atheromatous embolization should be considered in patients with simultaneous or sequential multisystem organ involvement. It is possible that the transient erosions seen in this patient were due to atheromatous embolization, however, this is not likely since there has been no recurrence of symptoms since bypass surgery. There has been no evidence at any time of peripheral vascular or renal involvement.

Ulceration of the stomach and duodenum has also been reported in association with occlusive vascular disease without evidence of atheromatous embolization. Force and colleagues (19), reported a patient with symptoms of mesenteric angina who, on endoscopy, had multiple superficial ulcers of the stomach and duodenum. The patient subsequently died and autopsy revealed extensive vascular disease but no evidence of cholesterol embolization.

\section{REFERENCES}

1. Parks DA, Jacobsen ED. Physiology of the splanchnic circulation. Arch Intern Med 1985; 145:1278-81.

2. Granger DN, Barrowman JA, Kvietys PR. Clinical Gastrointestinal Physiology. Philadelphia: WB Saunders, 1985:201-5.

3. Lanciault G, Jacobsen E. The gastrointestinal circulation. Gastroenterology 1976;71:851-73.

4. Perry MA, Ardell JL, Barrowman JA, Kvietys PR. Physiology of the splanchnic circulation. In: Kvietys PR, Barrowman JA, Granger DN, eds. Pathophysiology of the Splanchnic Circulation, Vol 1. Boca Raton: CRC Press, 1987:1-56.

5. Shanbour LL, Jacobsen ED. Digitalis and the mesenteric circulation. Dig Dis 1972;17:826-8. (Edit)

6. Anon. Chronic intestinal ischemia. $\mathrm{Br}$ Med J 1981;282:173. (Edit)
Garisch and Marks (20) also reported an elderly patient with a three month history of post prandial abdominal pain. Endoscopy showed extensive mucosal changes with gastric ulceration and duodenitis. Their patient died suddenly the day after endoscopy. Autopsy documented occlusion of all three splanchnic vessels with no mention of cholesterol embolization.

A third case was reported in 1982 by Allende and Ona (21). Their patient also presented with symptoms of chronic mesenteric ischemia. Multiple small mucosal erosions and a small ulcer in the gastric antrum with duodenal hyperemia were seen on endoscopy. The patient subsequently developed hepatic infarction and ischemic cholecystitis and died following a revascularisation procedure. The report does not mention the presence of cholesterol clefts at autopsy.

Talansky (22) reported multiple aphthous ulcers of the stomach, small bowel and colon in a 41-year-old man. At surgery the celiac, SMA and IMA were occluded. Their patient underwent bypass surgery and did well postoperatively. As in the present case, the presumptive diagnosis was that of Crohn's disease.

More recently, Hojgaard and Krag (23) reported a case very similar to the present one. A 54-year-old man presented with a history of chronic mesenteric ischemia. Upper endoscopy showed multiple small ulcers of the stomach, a chronic

7. Ottinger LW. Mesenteric ischemia. N Engl J Med 1982;307:535-7.

8. Marston A. Ischemia. Clin Gastroenterol 1985; 14:847-62.

9. Reiner L, Jiminez FA, Rodriguez FL. Atherosclerosis in the mesenteric circulation, Observations and correlations with aortic and coronary atherosclerosis. Am Heart ] 1963:66:200-9

10. Gorton M, John JF Jr. Polyarteritis overlap syndromes with extensive bowel infarction. Am J Gastroenterol 1980:74:153-6.

11. Nygaard K. Flatmark A. Mesenteric arteritis with extensive intestinal gangrene. Acta Clin Scand 1973;139:470-5.

12. Goulet Y, MacKay CG. Atheromatous embolisation: An entity with a polymorphous symptomatology. Can Med Assoc] 1963;88:1067-70. ulcer in the pyloric ring and severe duodenitis. The patient subsequently required emergency laparotomy and an SMA bypass was performed. Postoperative endoscopic resolution of ulceration was documented. At the time of writing this patient was well four years after surgery.

The finding of ischemic erosions in the superior mesenteric circulation would appear to carry a grave prognosis. Of the five patients reported thus far, two died before surgery could be performed and three required emergency laparotomy of whom one died post surgery. Following the demonstration of ischemic erosions the time to development of acute intestinal ischemia with subsequent death or emergency surgery varied from one day to six months $(20,21)$. In the present patient, the time from detection of the erosions to surgery was two months.

It is likely, however, that ischemic erosions occur more frequently than the volume of literature would suggest. Clearly, as demonstrated by this report, the erosions may be transient and missed at a single endoscopy.

The case reported here is unusual in many respects. To the authors' knowledge, ischemic erosions which have resulted spontaneously have not previously been reported. The case is also unusual in that an aberrant hepatic artery was probably of significance in precipitating the symptoms and signs.

13. Anderson WR, Richards AM, Weiss L. Hemorrhage and necrosis of the stomach and bowel due to atheroembolism. Am J Clin Pathol 1967:48:30-8.

14. Harvey RL, Dobernack RC, Black WC. Infarction of the stomach following atheromatous embolization. Gastroenterology 1972;62:469-72.

15. Bourdages R, Prentice RSA, Beck IT, et al. Atheromatous embolisation to the stomach. Dig Dis 1976;21:889-93.

16. Taylor NS, Gueft B, Lebowich RJ. Atheromatous embolisation: A cause of gastric ulcers and small bowel necrosis. Case report and review of the literature. Gastroenterology 1964:47:97-103.

17. Probstein JG, Joshi RA, Blumenthal HT. Atheromatous embolisation. An etiology of acute pancreatitis. Am Med Assoc Arch Surg 1957:75:566-72.

18. Sayre GP, Campbell DC. Multiple peripheral emboli in atherosclerosis of 
the aorta. Arch Intern Med

1959:103:799-806.

19. Force T, MacDonald BA, Eade OE, et al. Ischemic gastritis and duodenitis. Dig Dis Sci 1980;25:307-10.

20. Garisch JAM. Marks IN. Mesenteric ischemia - A diagnostic triad? S Afr

Med J 1980;57:91-2.

21. Allende HD, Ons FV. Celiac artery and superior mesenteric artery insufficiency. Gastroenterology 1982;82:763-6.

22. Talansky AL, Katz S, Naidich ].
Aphthous ulcers in ischemic gastroenterocolitis: A case report. Am ] Gastroenterol 1985;80:257-9

23. Hojgaard L, Krag E. Chronic ischemic gastritis reversed after operation. Gastroenterology 1987;92:226-8. 


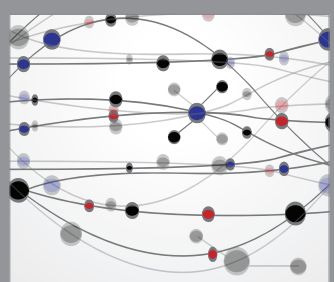

The Scientific World Journal
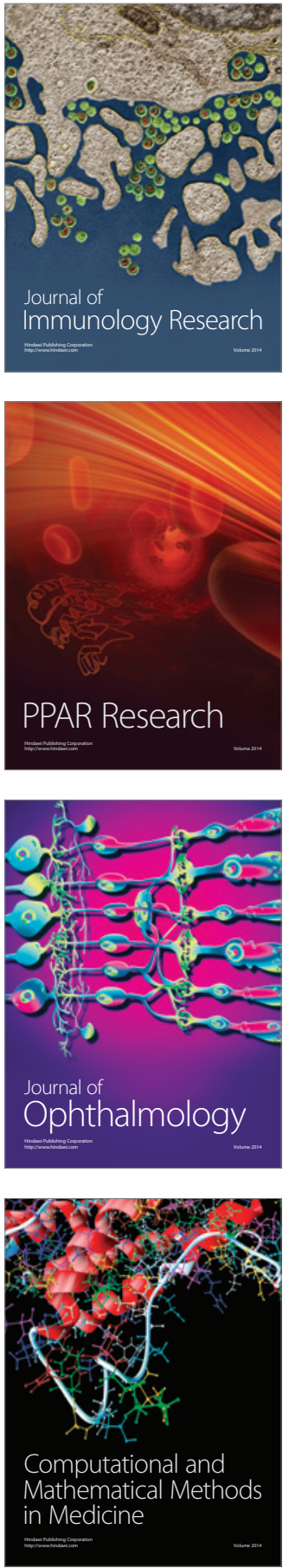

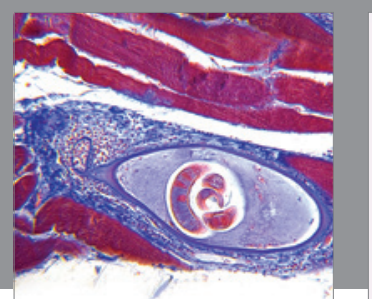

Gastroenterology Research and Practice

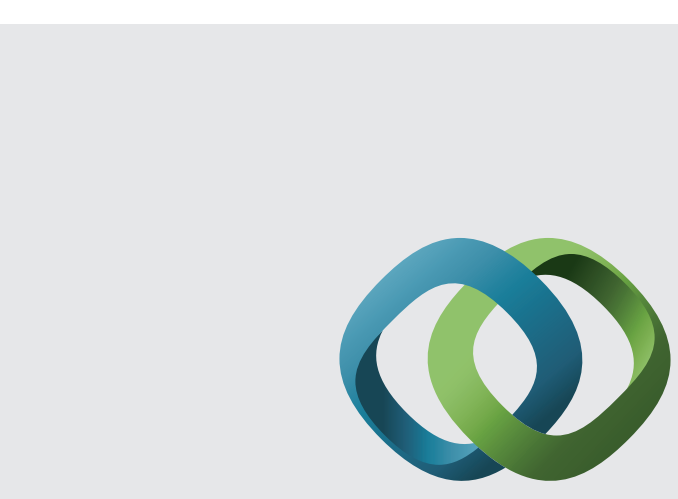

\section{Hindawi}

Submit your manuscripts at

http://www.hindawi.com
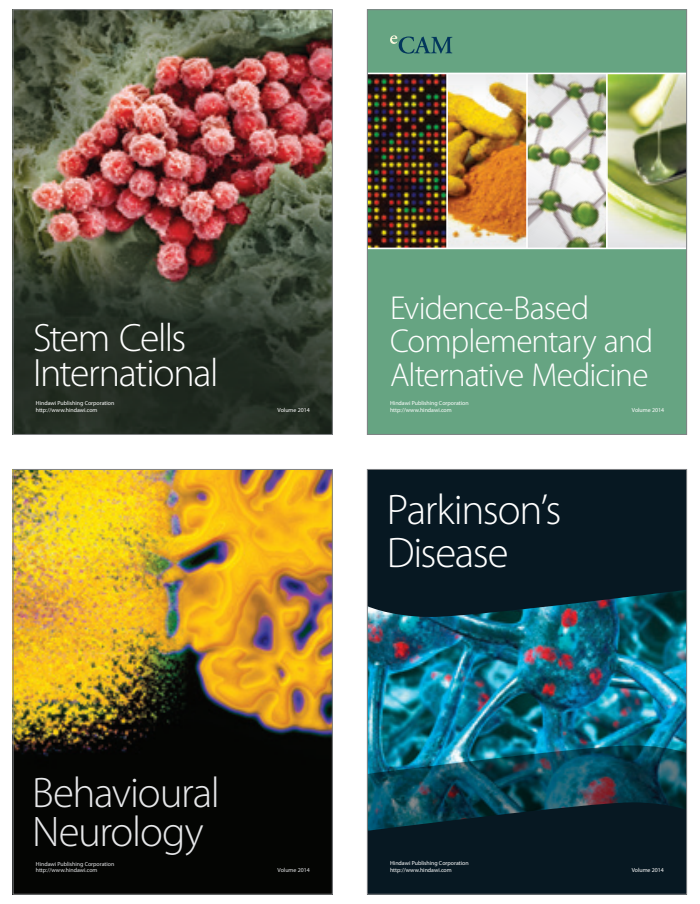
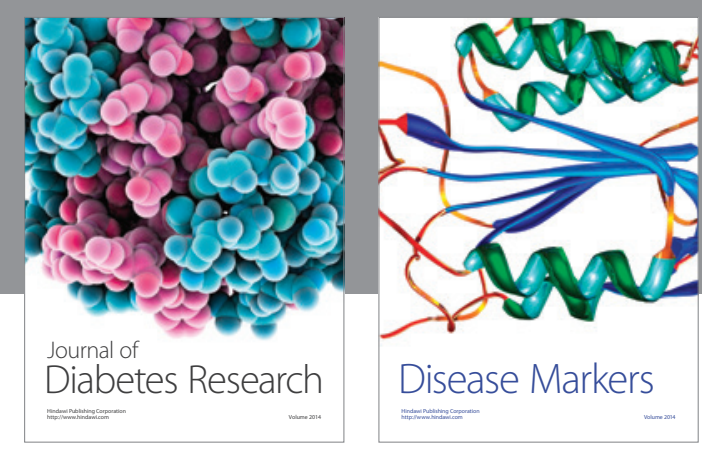

Disease Markers
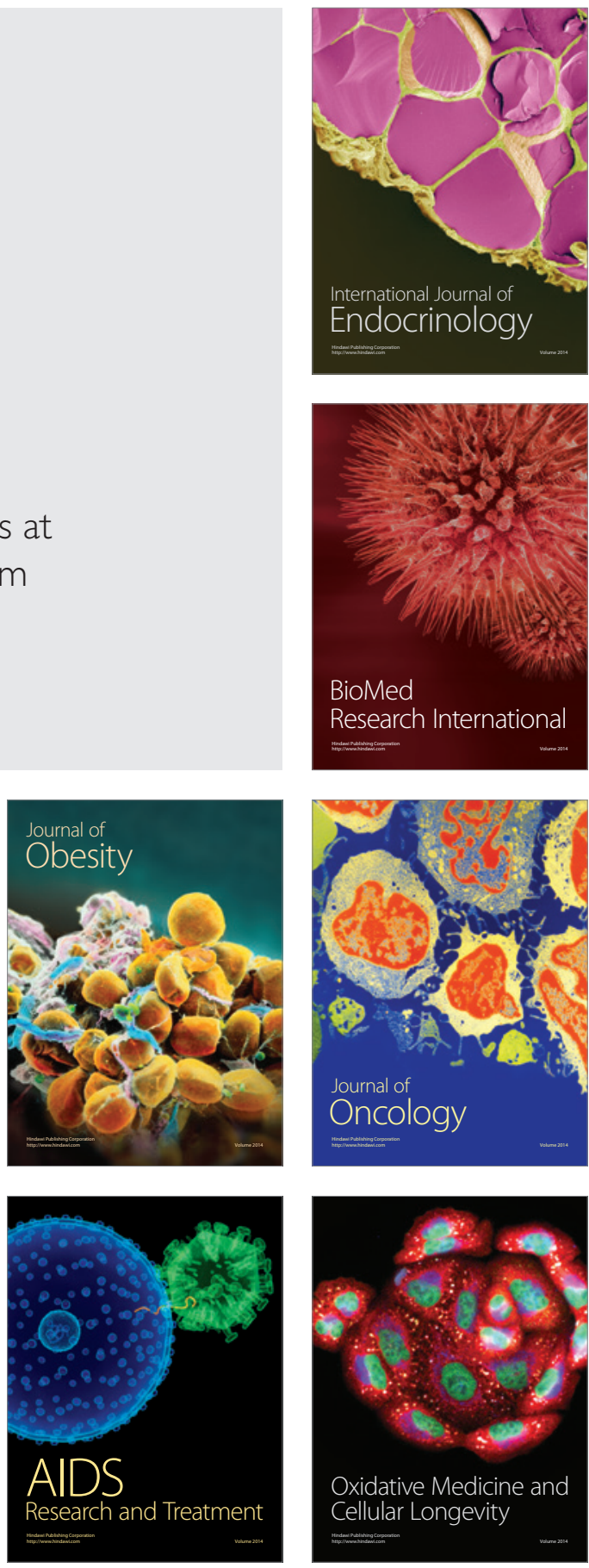